M. TANIGUCHI KODAI MATH. J.

10 (1987), $165-173$

\title{
A NOTE ON CONTINUITY OF GREEN'S FUNCTIONS ON RIEMANN SURFACES
}

\author{
BY MASAHIKO TANIGUCHI
}

\section{$\S 1$. Introduction and main results.}

Let $R$ be an arbitrary Riemann surface admitting Green's functions, and denote by $g(\cdot, r)$ Green's function with the pole $r \in R$. Also set $U(r, M)=$ $\{s \in R: g(s, r)>M\}$ for every $r \in R$ and positive $M$. Then we have the following

ThEOREM 1. Let $q$ be a point on $R$. Take a positive constant $M$ so large that $U(q, M)$ is simply connected. Then it holds that

$$
\left\|d g\left(\cdot, q^{\prime}\right)-d g(\cdot, q)\right\|_{R-U(q, M)}<6 \cdot e^{M+4} \cdot \exp \left(-g\left(q^{\prime}, q\right)\right)
$$

for every $q^{\prime} \in U(q, M+4)$.

Theorem 1 is a corollary of Lemma 2 in $\S 2$, which also gives the following

THEOREM 2. Under the same assumptions as in Theorem 1, it holds that

$$
\left|\int_{d} * d g\left(\cdot, q^{\prime}\right)-\int_{d} * d g(\cdot, q)\right| \leqq 9\left(\lambda_{d}\right)^{1 / 2} \cdot e^{M+4} \cdot \exp \left(-g\left(q^{\prime}, q\right)\right)
$$

for every 1-cycle $d$ on $R-U(q, M+4)$ and $q^{\prime} \in U(q, M+4)$. where $\lambda_{d}$ is the extremal length of the homology class of $d$ on $R$.

THEOREM 3. Let $p$ and $q$ be two distinct points on $R$. Take a positive $M$ so large that $U(q, M)$ is contained in $R-\{p\}$ and simply connected. Then it holds that

$$
\begin{aligned}
\left|g\left(q^{\prime}, p\right)-g(q, p)\right| & \leq 5(g(q, p))^{1 / 2} \cdot e^{M+4} \cdot \exp \left(-g\left(q^{\prime}, q\right)\right) \\
& \leq 5 M^{1 / 2} \cdot e^{M+4} \cdot \exp \left(-g\left(q^{\prime}, q\right)\right)
\end{aligned}
$$

for every $q^{\prime} \in U(q, M+4)$.

The proof of Theorems 1 and 2,3 are given in $\S 1$ and $\S 2$, respectively. Here we note the following corollary of Theorem 3 .

COROLlaRY. Let $R$ be a Riemann surface satısfying the following condition;

Received July 28, 1986 
(*) there is a positive constant $M$ such that $U(q, M)$ is simply connected for every $q \in R$.

Then Green's functions are locally unformly Lipschitz-like contınuous, i.e. it holds that

$$
\left|g\left(q_{1}, p\right)-g\left(q_{2}, p\right)\right| \leq 5 M^{1 / 2} \cdot e^{M+4} \cdot \exp \left(-g\left(q_{1}, q_{2}\right)\right)
$$

for every $q_{1}$ and $q_{2}$ in $R-\overline{U(p, M)}$ such that $g\left(q_{1}, q_{2}\right)>M+4$.

Next combining Theorem 3 with the comparison theorem in [4], we can show, in general, the following local Lipschitz-like continuity of Green's functions.

THEOREM 4. Under the same assumption as in Theorem 3, it holds that

$$
\left|g\left(q_{1}, p\right)-g\left(q_{2}, p\right)\right| \leq C_{1} \cdot(M+1)^{1 / 2} \cdot e^{M} \cdot \exp \left(-g\left(q_{1}, q_{2}\right)\right)
$$

for every $q_{1}$ and $q_{2}$ in $U\left(q, M+C_{0}\right)$, where $C_{0}$ and $C_{1}$ are suitable absolute constants.

In case that $g(q, p)$ is sufficeintly large, or equivalently $q$ is sufficiently near to $q$, we can show the following

THEOREM 5. Let $p$ and $q$ be distinct points on $R$ such that $M=g(q, p)>C_{2}$ and $U\left(q, M-C_{2}\right)$ is simply connected with a suitable absolute constant $C_{2}$. Then it holds that

$$
\left|g\left(q_{1}, p\right)-g\left(q_{2}, p\right)\right| \leq C_{3} \cdot e^{M} \cdot \exp \left(-g\left(q_{1}, q_{2}\right)\right)
$$

for every $q_{1}$ and $q_{2}$ in $U\left(q, M+C_{2}\right)$ with a suitable absolute constant $C_{3}$.

The proofs of Theorems 4 and 5 are given in $\S 4$. And finally as an application of Theorem 1, we will include in $\S 5$ a remark to the remainder terms of variational formulas in [5].

\section{$\S 2$. The proof of Theorem 1 .}

Let $g^{*}(s)$ be a conjugate harmonic function of $g(s, q)$ on $U(q, M)$, and set $z=Z(s)=e^{M+2} \cdot \exp \left(-g(s, q)-i \cdot g^{*}(s)\right)$. Then $Z(U(q, M+n))=U\left(e^{2-n}\right)$ for every non-negative $n$, where we set $U(\rho)=\{z:|z|<\rho\}$ for every positive $\rho$. For every $a$ in $U(1 / e)$, define a mapping $f_{a}$ of $R$ onto itself by setting

$$
\begin{aligned}
Z \circ f_{a} \circ Z^{-1}(z) & =z+a \quad \text { on } U(1), \text { and } \\
& =(a|z|+z) \cdot(1+a(|z| / z))^{-10 g|z|}
\end{aligned}
$$

on $U(e)-\overline{U(1)}$, and by letting $f_{a}$ be the identical mapping on $R-U(q, M+1)$, where we choose the branch of $\log (1+a(|z| / z))$ so that $\log 1=0$. 
Note that $f_{a}$ is conformal outside of $\left.W=\overline{U(q, M+1}\right)-U(q, M+2)$, and we can show the following

LEMMA 1. If $|a|<(e-2) / e(<1 / e)$, then $f_{a}$ is $\left(1+k_{a}\right) /\left(1-k_{a}\right)$-quasiconformal on $R$ with $k_{a} \leq e \cdot|a| /(e-2)$.

Proof. Set $F(z)=Z \circ f_{a} \circ Z^{-1}(z)$ on $U(e)-\overline{U(1)}$, then by a simple computation we have

$$
\begin{aligned}
& \frac{F_{z}}{F}=\frac{1}{2 z}\left(2-(1-\log |z|) \cdot \frac{a|z|}{z+a|z|}-\log \left(1+a \frac{|z|}{z}\right)\right), \quad \text { and } \\
& \frac{F_{\bar{z}}}{F}=\frac{1}{2 \bar{z}}\left((1-\log |z|) \cdot \frac{a|z|}{z+a|z|}-\log \left(1+a \frac{|z|}{z}\right)\right)
\end{aligned}
$$

Since $|a|<1 / e$, it holds that

$$
\begin{aligned}
& \left|\frac{a|z|}{z+a|z|}\right| \leq \frac{|a|}{1-|a|} \leq e|a| /(e-1)<1 /(e-1), \quad \text { and } \\
& |\log (1+a(|z| / z))| \leq|a| \sum_{n=0}^{\infty}|a|^{n}<e|a| /(e-1)<1 /(e-1) .
\end{aligned}
$$

Hence we have

$$
\left|F_{\bar{z}} / F_{z}\right| \leq\left(\frac{2 e}{e-1} \cdot|a|\right) /(2-2 /(e-1)) \leq e|a| /(e-2) \quad(<1) .
$$

q.e.d.

Now fix $q^{\prime} \in U(q, M+4)$ and set $a=Z\left(q^{\prime}\right)$. Then $|a|=e^{M+2} \cdot \exp \left(-g\left(q^{\prime}, q\right)\right)<$ $1 / e^{2}<(e-2) / e$. Writting $\varphi_{r}=d g(\cdot, r)+i^{*} d g(\cdot, r)$ for every $r \in R$, we set $\omega=$ $\varphi_{q^{\prime}} \circ f_{a}-\varphi_{q}$, where $\varphi_{q^{\prime}} \circ f_{a}$ is the pull-back of $\varphi_{q^{\prime}}$ by $f_{a}$. Then we know the following lemma, which implies the assertion of Theorem 1 (cf. [1, Theorem 5], [3, Proposition 5]).

LEMMA 2. It holds that

$$
\|\omega\|_{R} \leq \frac{\sqrt{2} \cdot k_{a}}{1-k_{a}}\left\|\varphi_{q}\right\|_{W}<6 \cdot e^{2} \cdot|a|,
$$

where $\|\alpha\|_{E}$ is the Dirichlet norm of $\alpha$ on a Borel set $E$.

Proof. For the sake of convenience, we include the proof. Since $\operatorname{Re} \omega \in$ $\Gamma_{e 0}(R)$ and $\operatorname{Im} \omega \in \Gamma_{c}(R)$, we have

$$
\iint_{R} \omega \wedge \bar{\omega}=2 i \cdot(\operatorname{Re} \omega, * \operatorname{Im} \omega)_{R}=0,
$$

where and in the sequel, $\Gamma(R)$ is the Hilbert space of real square integrable differentials on $R, \Gamma_{c}(R)$ and $\Gamma_{h}(R)$ are subspaces of $\Gamma(R)$ consisting of closed 
and harmonic differentials, respectively, $\Gamma_{e 0}(R)$ is the orthogonal complement of $\Gamma_{h}(R)$ in $\Gamma_{c}(R)$, and we set $(\alpha, \beta)_{E}=\iint_{E} \alpha \wedge * \beta$ for every $\alpha$ and $\beta$ in $\Gamma(R)$ and $E$ as above. by $(*)$

Writting $\varphi_{q^{\prime}}=g(w) d w$ with a generic local parameter $w$ on $R$, we have

$$
\begin{aligned}
& \left\|g \circ f_{a} \cdot\left(f_{a}\right)_{w} d w-\varphi_{q}\right\|_{W} \leq\left\|g \circ f_{a} \cdot\left(f_{a}\right)_{w} d w-\varphi_{q}\right\|_{R} \\
= & \left\|g \circ f_{a} \cdot\left(f_{a}\right)_{\bar{w}} d \bar{w}\right\|_{R} \leq k_{a} \cdot\left\|g \circ f_{a} \cdot\left(f_{a}\right)_{w} d w\right\|_{W},
\end{aligned}
$$

which implies that

$$
\left\|g \circ f_{a} \cdot\left(f_{a}\right)_{w} d w\right\|_{W} \leq \frac{1}{1-k_{a}}\left\|\varphi_{q}\right\|_{W}
$$

Thus we have

$$
\begin{aligned}
\|\omega\|_{R}^{2} & =\left\|g \circ f_{a} \cdot\left(f_{a}\right)_{w} d w-\varphi_{q}\right\|_{R}^{2}+\left\|g \circ f_{a} \cdot\left(f_{a}\right)_{\bar{w}} d \bar{w}\right\|_{R}^{2} \\
& \leq 2\left(k_{a}\right)^{2} \cdot\left\|g \circ f_{a} \cdot\left(f_{a}\right)_{w} d w\right\|_{W}^{2} \leq 2\left(k_{a} /\left(1-k_{a}\right)\right)^{2} \cdot\left\|\varphi_{q}\right\|_{W}^{2},
\end{aligned}
$$

which shows the first inequality. Next, since $\left\|\varphi_{q}\right\|_{W}^{2}=4 \pi$ and $\left|k_{a}\right| \leq e \cdot|a| /(e-2)$ $<1 / e(e-2)$ by Lemma 1 , we can see the second inequality.

\section{$\S 3$. The proofs of Theorems 2 and 3 .}

Theorems 2 and 3 follows from Lemma 2 by recalling the following facts (cf. $[2, \S 3]$ ). Again for the sake of convenience, we include their proofs.

LEMMA 3. Under the same assumptions as in Theorem 2, it holds that

$$
\int_{d} * d g\left(\cdot q^{\prime}\right)-\int_{d} * d g(\cdot, q)=-\operatorname{Re} \iint_{R} \omega \wedge \theta_{d},
$$

where, letting $\sigma_{d}$ be the differential in $\Gamma_{h}(R)$ such that $\left(\alpha, \sigma_{d}\right)_{R}=\int_{d} \alpha$ for every $\alpha \in \Gamma_{h}(\boldsymbol{R})$, we set $\theta_{d}=\sigma_{d}+i^{*} \sigma_{d}$.

Proof. Since $\operatorname{Re} \omega \in \Gamma_{e 0}(R)$ and $\operatorname{Im} \omega \in \Gamma_{c}(R)$, we have $\left(\operatorname{Re} \omega, * \sigma_{d}\right)_{R}=0$ and $\left(\operatorname{Im} \omega, \sigma_{d}\right)_{R}=\int_{d} \omega$, which implies the assertion.

q.e.d.

LEMMA 4. Under the same assumptions as in Theorem 3, it holds that

$$
g\left(q^{\prime}, p\right)-g(q, p)=\frac{1}{2 \pi} \cdot \operatorname{Re} \iint_{R} \omega \wedge^{*} \varphi_{p} .
$$

Proof. Since $U(q, M) \boxplus p$, we can find a positive $N$ so large that $U(p, N-1)$ is simply connected and disjoint from $U(q, M+1)$. Fix such an $N$, and let $J(s)$ 
be a smooth function on $R$ such that $J(s) \equiv 1$ on $U(p, N-1 / 2)$ and $J(s) \equiv 0$ on $R-U(p, N-1)$. Set

$$
\omega_{1}=d\left((1-J(\cdot)) \cdot\left(g\left(f_{a}(\cdot), q^{\prime}\right)-g(\cdot, q)\right)\right),
$$

then we have $\omega_{1} \in \Gamma_{e 0}\left(R_{1}\right)$ with $R_{1}=R-\overline{U(p, N)}$. Hence by Green's formula, we have

$$
\begin{aligned}
& \left(\operatorname{Re} \omega, \operatorname{Re} \overline{\varphi_{p}}\right)_{R_{1}}=\left(\operatorname{Re} \omega-\omega_{1}, d g(\cdot, p)\right)_{R_{1}} \\
& =\int_{-\partial U(p, N)}\left(g\left(\cdot, q^{\prime}\right)-g(\cdot, q)\right) \cdot * d g(\cdot, p)=2 \pi\left(g\left(p, q^{\prime}\right)-g(p, q)\right) .
\end{aligned}
$$

Similarly we can see that

$$
\begin{aligned}
\left(\operatorname{Im} \omega, \operatorname{Im} \overline{\varphi_{p}}\right)_{R_{1}} & =\int_{-\partial U(p, N)}-g(\cdot, p) \cdot \operatorname{Im} \omega \\
& =N \int_{-\partial U(p, N)}-\operatorname{Im} \omega=0 .
\end{aligned}
$$

Finally since $\omega$ is holomorphic on $U(p, N)$, it holds that $\iint_{U(p, N)} \omega \wedge^{*} \varphi_{p}=0$, and we have the desired equation.

Proof of Theorem 2. By Lemma 3, we have

$$
\left|\int_{d} * d g\left(\cdot, q^{\prime}\right)-\int_{d} * d g(\cdot, q)\right| \leq\left|\iint_{R} \omega \wedge \theta_{d}\right| \leq\|\omega\|_{R} \cdot\left\|\theta_{d}\right\|_{R} .
$$

Since $\left\|\theta_{d}\right\|_{R}^{2}=2 \lambda_{d}$ by Accola's theorem, we conclude the assertion by Lemma 2 . q.e.d.

Proof of Theorem 3. Since $\omega \wedge^{*} \varphi \equiv 0$ on $R-\bar{W} \cup\{p\}$, we have

$$
\begin{gathered}
\left|g(q, p)-g\left(q^{\prime}, p\right)\right| \leq(1 / 2 \pi) \cdot\left|\iint_{R} \omega \wedge^{*} \varphi_{p}\right| \\
=(1 / 2 \pi) \cdot\left|\iint_{W} \omega \wedge^{*} \varphi_{p}\right| \leq(1 / 2 \pi) \cdot\|\omega\|_{R} \cdot\left\|\varphi_{p}\right\|_{W}<e^{2} \cdot\left\|\varphi_{p}\right\|_{W} \cdot|a| .
\end{gathered}
$$

Next since $W$ is contained in $U(q, M+1)$ and $g(\cdot, p)$ is positive harmonic on $U(q, M)$, Harnack's inequality implies that

$$
\sup _{s \in W} g(s, p)-\inf _{s \in W} g(s, p) \leq \frac{4 e}{e^{2}-1} \cdot g(q, p) .
$$

Here recall that $\|d g(\cdot, p)\|_{R-U(p, N)}^{2}=\int_{-\partial U(p, N)} g(\cdot, p)^{*} d g(\cdot, p)=2 \pi N$ for every sufficiently large $N$ (cf. the proof of Lemma 4). And since $g(\cdot, p)-t$ is Green's function on $\{r \in R: g(r, p)>t\}$ for every positive $t$, we can see that $\|d g(\cdot, p)\|_{\left.i r \in R: t<g(r, p)<t^{\prime}\right)}^{2}=2 \pi\left(t^{\prime}-t\right)$ for every $t$ and $t^{\prime}$ with $0<t<t^{\prime}$. Hence we conclude that 


$$
\left\|\varphi_{p}\right\|_{W}^{2} \leq 4 \pi \cdot \frac{4 e}{e^{2}-1} \cdot g(q, p)<25 g(q, p),
$$

which gives that

$$
\left|g\left(q^{\prime}, p\right)-g(q, p)\right|<5 e^{2} \cdot g(q, p)^{1 / 2} \cdot|a| .
$$

The second inequality follows by recalling that $g(q, p) \leq M$, for $p \oplus U(q, M)$. q. e. d.

Remark. The author guess that, in case that $g(q, p)<1$, we can show that $\left\|\varphi_{p}\right\|_{W} \leq A \cdot(g(q, p))^{\lambda}$ with some $\lambda>1 / 2$ and a constant $A$ (which may depend on $p$ and $R$ ). Note that such $\lambda$ should not be greater than 1 .

\section{$\S 4$. The proofs of Theorems 4 and 5 .}

For the proofs, the following lemma is crucial.

LEMma 5. There is an absolute constant $C$ such that, for every $q$ and $M$ as in Theorem 1, and every $q^{\prime} \in U(q, M+C)$, we can find an $M\left(q^{\prime}\right)$ such that $M \leq$ $M\left(q^{\prime}\right) \leq M+C, U\left(q^{\prime}, M\left(q^{\prime}\right)\right)$ is simply connected and

$$
U(q, M+C)<U\left(q^{\prime}, M\left(q^{\prime}\right)\right)<M(q, M) .
$$

Proof. Set $C=B+1$ with an absolute constant $B$ in [4, Proposition 2], and apply [4, Proposition 2] to $h(z)=(1 / 2 \pi) \cdot g\left(Z^{-1}(z), q^{\prime}\right)$ on $Z\left(W_{0}\right)$ with $W_{0}=$ $U(q, M+1 / 4)-\overline{U(q, M+C-1 / 4)}$. Then we have an $M\left(q^{\prime}\right)$ such that $\{s \in R$ : $\left.g\left(s, q^{\prime}\right)=M\left(q^{\prime}\right)\right\}$ is a simple closed curve in $W_{0}$ separating two boundary components of $W_{0}$.

Fix such an $M\left(q^{\prime}\right)$, then it is clear that $U\left(q^{\prime}, M\left(q^{\prime}\right)\right)$ is simply connected and satisfies (I). And since, in general, $g(\cdot, r) / M$ is the harmonic measure of $\partial U(r, M)$ in $R-\overline{U(r, M)}$, for every $r \in R$ and positive $M$, we can see that $M \leq$ $M\left(q^{\prime}\right) \leq M+C$.

q. e. d.

Proof of Theorem 4. By Lemma 5, we can apply Theorem 3 with $q_{1} \in$ $U(q, M+C)$ and $M+C$. Then we have

$$
\begin{aligned}
\left|g\left(q_{2}, p\right)-g\left(q_{1}, p\right)\right| & \leq 5 \cdot(M+C)^{1 / 2} \cdot e^{M+C+4} \cdot \exp \left(-g\left(q_{1}, q_{2}\right)\right) \\
& \leq 5 C \cdot e^{C+4}(M+1)^{1 / 2} \cdot e^{Y} \cdot \exp \left(-g\left(q_{1}, q_{2}\right)\right)
\end{aligned}
$$

for every $q_{2}$ in $U\left(q_{1}, M+C+4\right)$.

On the other hand, if $q_{1} \in U(q, M+2 C+4)$, then $q \in U\left(q_{1}, M+2 C+4\right)$ by symmetry, and hence again by Lemma 5 we see that $U(q, M+2 C+4)$ is contained in $U\left(q_{1}, M+C+4\right)$. Hence the assertion holds with $C_{0}=2 C+4$ and $C_{1}=5 C \cdot e^{C+4}$.

q. e. d.

Next to show Theorem 5, we need the following 
LEMMA 6. Let $p$ and $q$ be distinct points on $R$ such that $U(q, M-C)$ is simply connected with $M=g(q, p)$ and $C$ given in Lemma 5 . Then it holds that

$$
\left|g\left(q^{\prime}, p\right)-g(q, p)\right| \leq C^{\prime} \cdot e^{M} \cdot \exp \left(-g\left(q^{\prime}, q\right)\right)
$$

for every $q^{\prime} \in U(q, M+C+4)$, where $C^{\prime}$ is an absolute constant.

Proof. By Lemma 5 we have

$$
U\left(q^{\prime}, M-C\right) \supset U(q, M) \supset U\left(q^{\prime}, M+C\right)
$$

for every $q^{\prime} \in U(q, M+C)$, which implies that

$$
\begin{aligned}
& \sup _{s \in U(q, M+C)} g(s, p) \leq M+C, \text { and } \\
& \inf _{s \in U(q, M+C)} g(s, p) \geq M-C .
\end{aligned}
$$

In particular, it holds that $\left\|\varphi_{p}\right\|_{U(q, M+C)} \leq 4 \pi \cdot 2 C$.

Hence by the same argument as in the proof of Theorem 3, we have

$$
\begin{aligned}
& \left|g\left(q^{\prime}, p\right)-g(q, p)\right|<e^{2} \cdot\left\|\varphi_{p}\right\|_{W} \cdot|a| \\
& \leq e^{M+C+4} \cdot(8 \pi C)^{1 / 2} \cdot \exp \left(-g\left(q^{\prime}, q\right)\right)
\end{aligned}
$$

for every $q^{\prime} \in U(q, M+C+4)$, i. e., the assertion holds with $C^{\prime}=e^{C+4} \cdot(8 \pi C)^{1 / 2}$.

q.e.d.

Proof of Theorem 5. Suppose that $U(q, M-3 C)$ is simply connected with $M=g(p, q)$. Then we can see by Lemma 5 that, for every $q_{1} \in U(q, M+C)$, it holds that $M-C \leq M_{1}=g\left(q_{1}, p\right) \leq M+C$ and $U\left(q_{1}, M-2 C\right)$ is simply connected. Hence by Lemma 6 we have

$$
\left|g\left(q_{2}, p\right)-g\left(q_{1}, p\right)\right|<C^{\prime} \cdot e^{M_{1}} \cdot \exp \left(-g\left(q_{1}, q_{2}\right)\right)
$$

for every $q_{2} \in U\left(q_{1}, M_{1}+C+4\right)$.

Thus as in the proof of Theorem 4 , we can show that $C_{2}=3 C+4$ and $C_{3}=$ $C^{\prime} \cdot e^{C}$ are desired constants.

q. e. d.

\section{§5. Another application of Theorem 1.}

In this section, we will use the same notation as in [5], and show that the remainder terms in the formulas (2) and (3) of [5, Theorem 2] can be estimated locally uniformly on $R_{0}^{\prime}$ with respect to $q$ and $q^{\prime}$. Here we will discuss only (3), for the treatment of (2) is the same.

Let $F\left(q, q^{\prime}, t\right)$ be the remainder term, i. e., 


$$
\begin{aligned}
F\left(q, q^{\prime}, t\right)= & g\left(q, q^{\prime} ; R_{t}\right)-g\left(q, q^{\prime} ; R_{0}\right)-\left\{-\frac{1}{2} \cdot \log \left(\frac{1}{t}\right) \cdot G(q) \cdot G\left(q^{\prime}\right)\right. \\
& \left.-t^{2} \cdot \operatorname{Re}\left[\eta \cdot\left(b_{0, q, 1}(0) \cdot b_{0, q^{\prime}, 2}(0)+b_{0, q, 2}(0) \cdot b_{0, q^{\prime}, 1}(0)\right)\right]\right\} .
\end{aligned}
$$

Then we want to show the following

Proposition. When $t$ tends to $0, F\left(q, q^{\prime}, t\right) / t^{2}$ converges to 0 locally uniformly on $R_{0}^{\prime}$ with respect to $q$ and $q^{\prime}$.

Proof. By the equation $[5, \S 4(14)]$, we can see (cf. $[5,293 p])$ that

$$
\begin{aligned}
F\left(q, q^{\prime}, t\right)=\frac{-1}{2 \pi} \cdot \operatorname{Re}[ & \sum_{n=1}^{\infty} c_{n, 1} \cdot \oint_{\left\{\left|z_{2}\right|=t_{0}\right\}}\left(b_{t, q, 2}\left(z_{2}\right)-b_{0, q, 2}\left(z_{2}\right)\right) \cdot\left(\eta t^{2} / z_{2}\right)^{n} d z_{2} \\
& +\sum_{n=1}^{\infty} c_{n, 2} \cdot \oint_{\left(\left|z_{1}\right|=t_{0}\right\}}\left(b_{t, q, 1}\left(z_{1}\right)-b_{0, q, 1}\left(z_{1}\right)\right) \cdot\left(\eta t^{2} / z_{1}\right)^{n} d z_{1} \\
& \left.+\sum_{n=2}^{\infty}\left(c_{n, 1} \cdot e_{n, 2}+c_{n, 2} \cdot e_{n, 1}\right)(2 \pi \sqrt{-1})\left(\eta t^{2}\right)^{n}\right]
\end{aligned}
$$

where $\phi(0, q)=\sum_{n=1}^{\infty} e_{n, j} z_{j}^{n-1} d z$, and $\phi\left(0, q^{\prime}\right)=\sum_{n=1}^{\infty} n c_{n, j} z_{j}^{n-1} d z$, on $\bar{U}_{j}\left(M_{0}\right)(j=1,2)$. Integrating over a suitable compact interval in $\left[t_{0}, \exp \left(-M_{0}\right)\right)$, we see that

$$
\begin{aligned}
\left|F\left(q, q^{\prime}, t\right)\right| \leq & A\left(t^{2} \cdot\|\phi(t, q)-\phi(0, q)\|_{E} \cdot\left\|\phi\left(0, q^{\prime}\right)\right\|_{U_{0}}\right. \\
& \left.+t^{4}\|\phi(0, q)\|_{U_{0}} \cdot\left\|\phi\left(0, q^{\prime}\right)\right\|_{U_{0}}\right)
\end{aligned}
$$

with a suitable compact set $E$ in $U_{0}=U_{1}\left(M_{0}\right) \cup U_{2}\left(M_{0}\right)$ and a constant $A$ depending only on $E$.

Here by Theorem 1 (or, as is well-known), $\left\|\phi\left(0, q^{\prime}\right)\right\|_{U_{0}}$ is continuous, hence locally bounded on $R_{0}^{\prime}$ (as a function of $q^{\prime}$ ). Hence the assertion follows from the following lemma.

q. e.d.

LEMma 7. Set $F_{t}(q)=\|\phi(t, q)-\phi(0, q)\|_{E}$. Then $F_{t}(q)$ converges to 0 locally uniformly on $R_{0}^{\prime}$ as $t$ tends to 0 .

Proof. First recall that $\lim _{t \rightarrow 0} F_{t}(q)=0$ for every $q \in R_{0}^{\prime}$ which follows by [5, Theorem 1]. And it suffices to show that, for every $q_{0} \in R_{0}^{\prime}$ and every positive $\varepsilon>0$, there is a neighborhood $V$ of $q_{0}$ in $R_{0}^{\prime}$ and a positive $T$ such that $F_{t}(q)<\varepsilon$ for every $q \in V$ and $t \in[0, T]$.

To show this, note that

$$
\begin{gathered}
F_{t}(q) \leq 2\left\|d g_{t}(\cdot, q)-d g_{t}\left(\cdot, q_{0}\right)\right\|_{E}+F_{t}\left(q_{0}\right) \\
+2\left\|d g_{0}(\cdot, q)-d g_{0}\left(\cdot, q_{0}\right)\right\|_{E}
\end{gathered}
$$


Since $g_{t}\left(\cdot, q_{0}\right)$ converges to $g_{0}\left(\cdot, q_{0}\right)$ uniformly, at least, in some neighborhood $V_{0}$ of $q_{0}$ by [5, Corollary 1], we can apply Theorem 1 with the same $M$ to both $g_{t}$ and $g_{0}$ for every sufficiently small $t$. Hence we conclude that there is a neighborhood $V_{1}\left(\subset V_{0}\right)$ of $q_{0}$ and a $T_{0}(>0)$ such that for every $t \in\left[0, T_{0}\right]$ and $q \in V_{1}$ we have

$$
\left\|d g_{t}(\cdot, q)-d g_{t}\left(\cdot, q_{0}\right)\right\|_{E} \leq C^{\prime \prime} \cdot \exp \left(-g_{t}\left(q, q_{0}\right)\right)
$$

with a constant $C^{\prime \prime}$ depending only on $M$, from which the assertion follows easily.

q.e.d.

\section{REFERENCES}

[1] Y. Kusunoki and F. Maitani, Variations of abelian differentials under quasiconformal deformations, Math. Z., 181 (1982), 435-450.

[2] F. MAITANI, Variations of meromorphic differentials under quasiconformal deformations, J. Math. Kyoto Univ., 24 (1984), 49-66.

[3] M. TANIGUCHI, On convergence of holomorphic abelian differentials on the Teichmüller spaces of arbitrary Riemann surfaces, ibid., 24 (1984), 305-321.

[4] M. TANiguchi, On the modulus of the free homotopy class of a simple closed curve on an arbitrary Riemann surface, Japanese J. Math., 12 (1986), 53-68.

[5] M. TANiguchi, Continuity of certain differentials on finitely augmented Teichmüller spaces and variational formulas of Schiffer-Spencer's type, Tohoku Math. J., 38 (1986), 281-295.

Department of Mathematics

KYOTO UNIVERSITY 